\title{
Immunohistochemical Expression of IDH1R132H in Astrocytic Tumours and its Association with Histopathological Grade, TP53 and EGFR Protein Expression.
}

\author{
Shashank Mishra ${ }^{1 *}, \mathrm{~K}$ R Rathi ${ }^{2}$, Divya Shelly ${ }^{1}$ and Reena Bharadwaj ${ }^{1}$ \\ ${ }^{1}$ Department of Pathology, Armed Forces Medical College, Pune, India \\ ${ }^{2}$ Department of Pathology, Command hospital (SC), Pune, India
}

\begin{abstract}
Background: Molecular studies have uncovered that histopathologically diagnosed Astrocytomas are genetically heterogeneous. Majority of Diffuse Astrocytomas (DA), Anaplastic Astrocytomas (AA) and secondary Glioblastoma multiforme (GBM) have concurrent mutations of IDH, TP53 and ATRX. Astrocytomas without IDH mutation have distinct genotype and poor prognosis. Primary GBM show EGFR amplification and lack IDH mutation.

Methods: We studied 95 Astrocytic tumours including 15 Pilocytic Astrocytoma (PA), 21 Diffuse Astrocytomas, 7 Anaplastic Astrocytomas and 52 GBM using tissue-microarray (TMA) to assess immunohistochemical expression of IDH1R132H, TP53 and EGFR protein in all four grades and the association between these three immunohistochemical markers. TMA blocks with core size 3.0 mm were constructed using manual tissue-microarrayer. IHC for IDH1R132H, TP53 and EGFR was performed.

Results: A 13\%(2/15) of PA showed IDH1R132H expression. TP53 and EGFR expression was not seen in any case. 52\%(11/21), 71\%(15/21) and $9 \%(2 / 21)$ of DA showed expression of IDH1, TP53 and EGFR respectively. Frequency of mutant IDH1, TP53 and EGFR in Anaplastic astrocytoma was $85 \%(6 / 7), 85 \%(6 / 7)$ and $0 \%(0 / 7)$. Among GBMs there was significant difference in IHC expression of IDH1 and EGFR. Primary GBMs show high EGFR expression of $70 \%(26 / 37)$ and low IDH1 expression of 21\%(8/37). Secondary GBM in contrast show higher IDH1 expression $80 \%(12 / 15)$ and low EGFR expression of $13 \%(2 / 15)$.
\end{abstract}

Conclusion: IDH and TP53 mutations are seen in majority of DA, AA and secondary GBM and are hallmarks of these tumours. Primary GBMs have distinct molecular pathway. They lack IDH, TP53 mutation and overexpress EGFR.

Keywords: IDH, Glioma, EGFR, TP53, Immunohistochemistry, Genotype

\section{Introduction}

Gliomas account for $70 \%$ of primary brain tumours in adults with a yearly incidence of 6 cases per 100,000. [1] The 2007 WHO classification of CNS tumours was based on histopathological features, histogenesis and immunohistochemical expression of lineage-specific proteins. This approach has changed in the 2016 update, which has brought in the concept of integrated diagnosis. Integrated diagnosis uses molecular pathology in addition to light microscopy to classify CNS tumours. ${ }^{[2]}$

Glial tumours are divided into two major classes on the basis of invasion into surrounding brain tissue. Diffuse gliomas infiltrate into the adjacent brain parenchyma and recur even after gross total resection. These tumours respond poorly to conventional chemotherapy and radiation and are thus incurable. Therefore, understanding of their molecular pathways to develop more effective targeted therapy is urgently needed. Targeting driver molecular aberrations has been a very promising advance in recent years in cancer therapeutics and development of targeted therapies is much needed for gliomas.

Advances in molecular genetics have revealed that histopathologically diagnosed diffusely infiltrating astrocytomas consist of two genetically different groups of tumours. The majority have concurrent mutations of IDH1/ IDH2, TP53 and ATRX.Concurrent IDH1/2 and TP53 mutations are now considered to be a genetic hallmark of diffuse astrocytomas. ${ }^{[3]}$

The molecular pathogenesis of Glioblastomas is different and majority ( $>90 \%$ ) arise de-novo without any evidence of a pre-existing lower-grade astrocytoma. These GBMs are known as primary GBMs and show a higher prevalence of EGFR gene amplification ( $>40 \%)$ and EGFR overexpression $(>60 \%)$. Secondary GBM, in contrast, arise from lower grade gliomas and are seen in younger patients in comparison to primary GBMs. ${ }^{[4]}$ 
EGFR gene amplification is thus one of the genetic hallmarks of GBM and a tumour showing EGFR amplification should be treated like GBM even if the WHO histologic criteria of necrosis and microvascular proliferation are not met. ${ }^{[5]}$ EGFR signalling pathway, therefore, is a potential target for the development of targeted therapies using antibodies, tyrosine kinase inhibitors (TKIs) or vaccines. ${ }^{[6]}$

With this knowledge, we studied the immunohistochemical expression of IDH1R132H, TP53 and EGFR protein as a surrogate marker of genetic mutations among all four grades of astrocytoma and the association between these mutations. This study will help to better understand the pathways of gliomagenesis and the utility of immunohistochemistry to diagnose, classify, prognosticate astrocytomas and predict response to therapy.

\section{Materials and Methods}

In this study, cases of astrocytomas diagnosed at our institute from 2011to 2016 were studied. FFPE tissue blocks were retrieved from the archives. H\&E slides were evaluated to ascertain the diagnosis and WHO grade of astrocytomas by an experienced neuropathologist. The clinical data pertaining to the cases was collected from the histopathology database and requisition forms sent with the specimen.

To construct tissue microarray (TMA) H\&E slide from each tissue donor block was used to identify the most representative tumour area. H\&E slide was overlaid on the donor block and the area of interest was marked. $3 \mathrm{~mm}$ diameter cylindrical tissue cores were taken from donor tissue blocks using a manual tissue microarrayer (IHC world, USA). These cylindrical tissue cores were arrayed on an empty steel mould containing a small amount of molten paraffin in a $4 \times 3$ grid with the tissue cores facing down. First tissue core from a different tissue (Liver, spleen) served as an orientation marker. When the cylindrical cores got adhered to the mould additional molten wax at 60 degrees $\mathrm{C}$ was poured from the side and a paraffin block containing eleven tissue cores from astrocytic tumours and one core for orientation was prepared.

For the purpose of immunostaining, 3-4 micron thick sections from TMA blocks were obtained on poly-L-lysine coated glass slides and were incubated overnight at $37^{\circ} \mathrm{C}$. High-temperature antigen retrieval was performed by autoclaving the sections in $0.01 \mathrm{M}$ citrate buffer $(\mathrm{pH} 6.0)$ at $121^{\circ} \mathrm{C}$ for 10 minutes. Sections were treated with two drops of $3 \%$ hydrogen peroxide of the universal staining kit for 15-20 minutes to block the endogenous peroxidases, followed by protein blocking using ultra block of the universal staining kit for 5 minutes.
Immunohistochemical staining was performed by LSAB technique (LSAB Kit, M/s Abcam, Germany) using mouse monoclonal antibodies to IDH1 $2 \mathrm{H} 9$ in a dilution of 1:100. (Clone-ab117976; M/s Abcam, Germany). Subsequently, the biotinylated anti-mouse secondary of the universal staining kit followed by streptavidin/horse radish peroxidases was applied to the sections. Finally, DAB chromogen concentrate diluted in the substrate were applied, and slides were continuously seen under the microscope till desired colour intensity was reached. Counterstaining was done by Harris hematoxylin for a period of 1 minute. A visual semi-quantitative grading scale was applied to assess the immunoreactivity. A strong cytoplasmic staining in $>10 \%$ oftumour cells for IDHR132Hwas scored positive.

For studying EGFR protein expressionprimary polyclonal rabbit anti-EGFR antibody in 1:50 dilution (M/s Biogenex, Fremont CA) was used and a strong cytoplasmic staining in $>50 \%$ tumour cells for $E G F R$ was considered positive. A weak diffuse background staining was taken as negative.

Primary ready to use mouse monoclonal anti-human p53 protein antibody; clone DO-7 (M/s Dako, Denmark) was used for studying TP53 protein expression and a strong nuclear staining of tumour cell nuclei in $>10 \%$ of tumour cells was taken as positive.

\section{Result}

A total of 95 cases of astrocytic tumours were studied which included 15 pilocytic astrocytomas $(15.8 \%), 21$ diffuse astrocytomas (22.1\%), 07 anaplastic astrocytomas (7.4\%) and 52 glioblastomas (54.7\%). Among glioblastomas 37 $(38.9 \%)$ were de-novo or primary glioblastomas and 15 (15.8\%) were secondary glioblastomas.

62 patients were male (65\%) and 33 were female (34.7\%). The age range was 10-75 years and the mean age was 20.9 years for pilocytic astrocytoma (PA), 40.6 years for diffuse astrocytoma (DA), 46 years for anaplastic astrocytoma (AA), 53.4 years for primary GBM and 49 years for secondary GBM.

Isocitrate dehydrogenase 1 (IDHR132H) expression: When IDH1R132Hexpression was compared among different grades of astrocytoma it was observed that only 2/15 cases (13\%) of PAwere IDH1 positive. 11/21 (52\%) cases of DA and $6 / 7$ cases (85\%) of AA showed IDH1 expression.Among glioblastomas, there was a significant difference in the pattern of IDH1 expression among primary and secondary glioblastomas. IDH1 expression was significantly higher in secondary GBM and $12 / 15$ cases $(80 \%)$ showed positive immunostaining. In contrast, only 
$8 / 37$ cases $(21 \%)$ of primary GBM were IDH1 positive (Table 1, figure 1A). There was a significant association between grade of astrocytoma and IDH1R132H expression (Fisher's exact test, p-value $<0.001$ ).

TP53 expression: The wild type or unmutated p53 gene is easily degraded and is therefore not detectable by IHC. On the other hand, mutant p53, which has lost its normal function of tumour suppressor gene resists degradation and accumulates in the nucleus. This mutated TP53 which is accumulated in the nucleus is detected as strong nuclear positivity in tumour cells. It was observed that none of the 15 cases of PA expressed TP53 (0\%). Among DA and AA TP53 expression was high and $15 / 21$ cases (71\%) of DA and $6 / 7(86 \%)$ of AA were positive for TP53 expression. In contrast to grade II and III astrocytic tumours, the expression of TP53 was significantly lower in primary GBM with only $3 / 37$ cases $(8.1 \%$ ) cases showing positivity. Secondary glioblastomas also had a lower percentage of TP53 positive cases with 4/15 (26.6\%) showing nuclear positivity (Table 1, figure 1B).

EGFR protein expression: EGFR expression was significantly higher in primary glioblastomas with $26 / 37$ (70.3\%) cases expressing EGFR protein. In contrast, only $2 / 21$ cases $(9.5 \%)$ of DA and $2 / 15(13.3 \%)$ cases of secondary glioblastoma expressed EGFR protein. None of the PA or AA showed EGFR expression (Table 1, figure 1C). A significant association was found between tumour grade and EGFR protein expression using Fisher's exact test with a p-value of $<0.05$.

Association of IDH1 with EGFR and TP53 protein expression: When expression of IDH1 was compared with EGFR, it was observed that there is an inverse relationship between IDH1 and EGFR expression (figure 3D). Among the subgroup of 39 astrocytomas which were IDH1 positive only 6 cases (15.4\%) showed EGFR protein expression. Whereas, among 30 astrocytomas which were positive for EGFR protein; IDH1 expression was seen in
6 cases $(20 \%)$. Using chi-square test a significant inverse association was found between IDH1 and EGFR protein expression.

In contrast to EGFR it was noted that there is a positive association between IDH1 and TP53 expression. In 39 astrocytomas which were IDH1 positive 17 cases $(43.6 \%)$ showed TP53 expression. Whereas, among the subgroup of 56 astrocytomas which were negative for IDH1, TP53 expression was seen in 6 cases $(10.7 \%)$.A significant association was seen between IDH1 and TP53 expression (chi-square test, p-value 0.021)

\section{Discussion}

Previous studies have noted that IDH mutation is an early event in the pathogenesis of diffusely infiltrating gliomas (Grade II and III). ${ }^{[7]}$ Normal cellular function of IDH is to protect cells from oxidative stress by producing NADPH. NADPH is used to generate reduced glutathione (GSH), which is the main antioxidant in the central nervous system. ${ }^{[8,9]} \mathrm{R} 132 \mathrm{H}$ mutation in IDH1 reduces its enzymatic activity to convert Isocitrate to $\boldsymbol{\alpha}$-KG. ${ }^{[10]}$ Mutated IDH1 also acquires a new enzymatic activity and converts $\boldsymbol{\alpha}-\mathrm{KG}$ to D-2-hydroxyglutarate (D-2HG) and utilises NADPH in the process. ${ }^{[11]}$ This in turn reduces the concentration of reduced glutathione and makes the cells more susceptible to free radical induced injury and DNA damage. There is evidence to suggest that $\mathrm{D}-2 \mathrm{HG}$ is also an oncometabolite and is directly involved in the pathogenesis of IDH mutated tumours. ${ }^{[12]}$ Astrocytomas further acquire mutations in tumour suppressor gene TP53 and ATRX gene during the course of malignancy. ${ }^{[13]}$

Secondary GBM progress from pre-existing lower grade gliomas and thus also tend to express these molecular alterations. ${ }^{[14]}$ Primary GBM, on the contrary arise from a different genetic pathway and often show EGFR amplification. ${ }^{[15]}$ This difference in molecular pathogenesis among various grades of astrocytomas manifests as differential immunohistochemical profile among various grades.

Table 1: Table showing immunohistochemical expression of IDH1R132H, TP53 and EGFR protein among different grades of astrocytomas.

\begin{tabular}{|l|c|c|c|c|c|c|c|}
\hline WHO Grade & Total cases & IDH + & $\%$ IDH + & TP53 + & $\%$ TP53 + & EGFR + & $\begin{array}{c}\% \text { EGFR } \\
+\end{array}$ \\
\hline GRADE I & 15 & 2 & $13 \%$ & 0 & $0 \%$ & 0 & $0 \%$ \\
\hline GRADE II & 21 & 11 & $52 \%$ & 15 & $71 \%$ & 2 & $9 \%$ \\
\hline GRADE III & 7 & 6 & $85 \%$ & 6 & $85 \%$ & 0 & $0 \%$ \\
\hline Primary GBM & 37 & 8 & $21 \%$ & 3 & $8 \%$ & 26 & $70 \%$ \\
\hline Secondary GBM & 15 & 12 & $80 \%$ & 4 & $27 \%$ & 2 & $13 \%$ \\
\hline
\end{tabular}




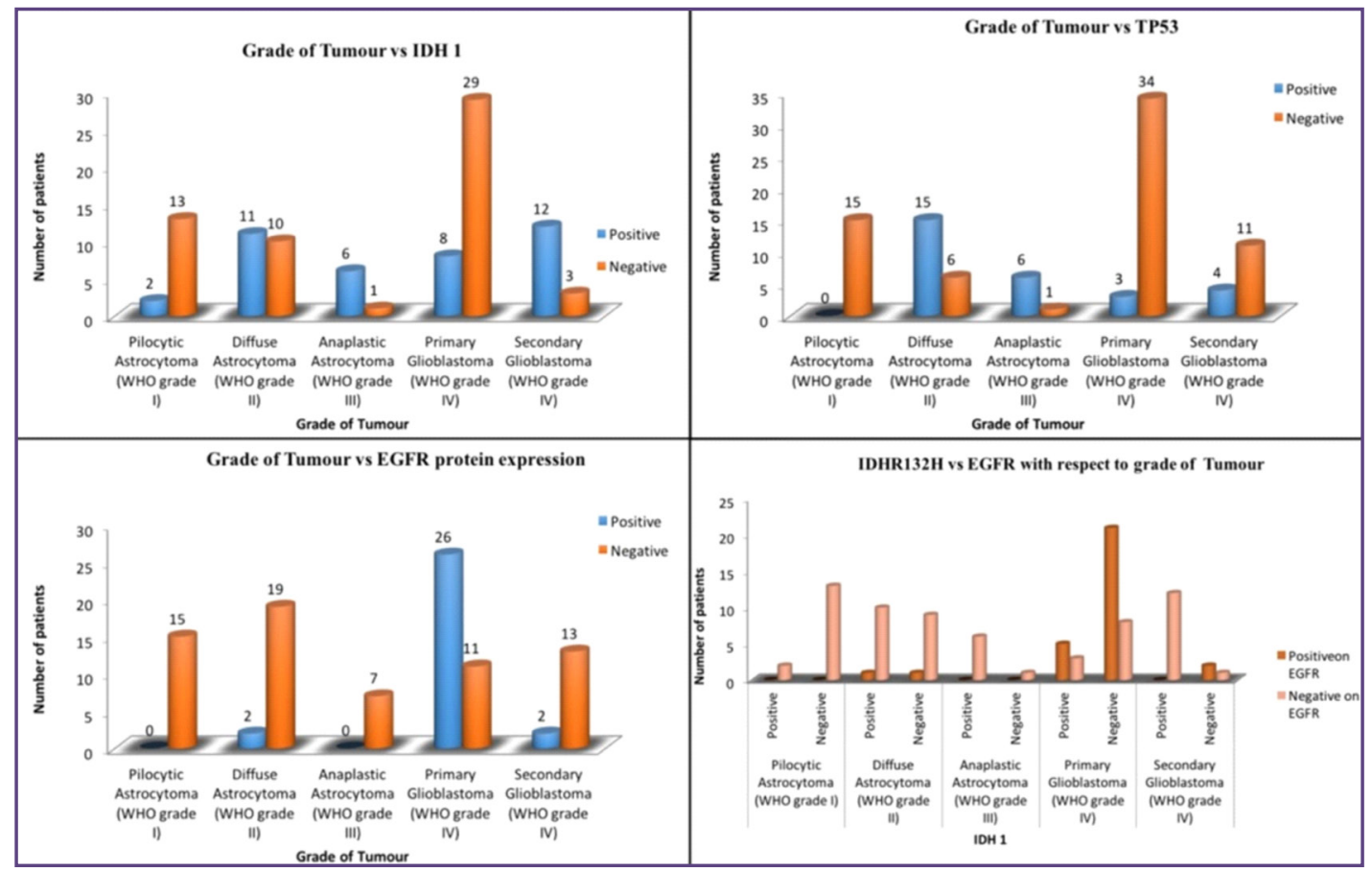

Fig. 1: Bar diagrams showing IDH1R132H (1A), TP53 (1B), EGFR (1C) expression among different grades of astrocytoma. Figure 1D shows association of IDH1 and EGFR expression.

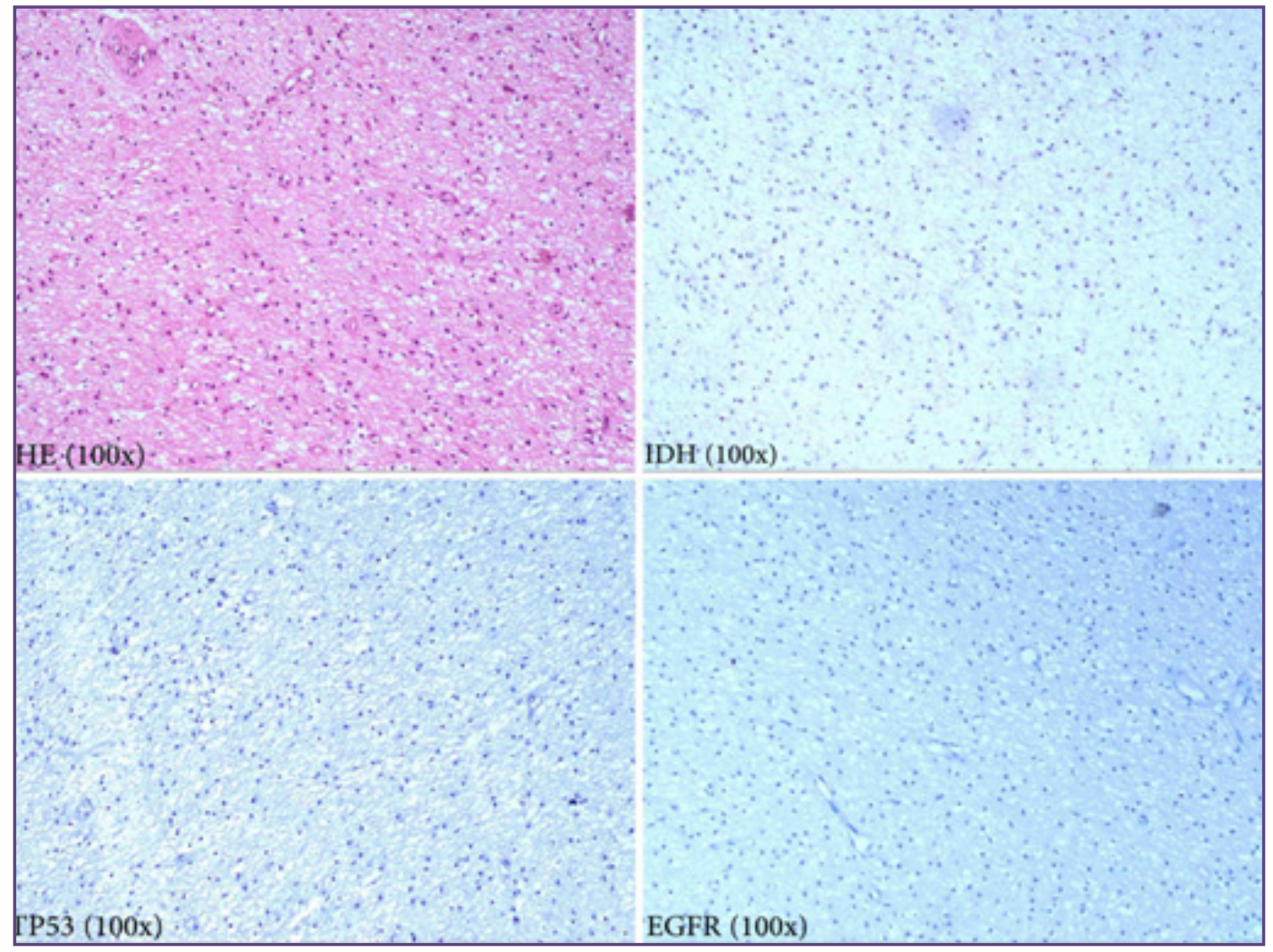

Fig. 2: Photomicrograph showing H\&E stained section of Pilocytic Astrocytoma (WHO grade I) with IDH1R132H, TP53 and EGFR protein expression. All three IHC markers are negative. 


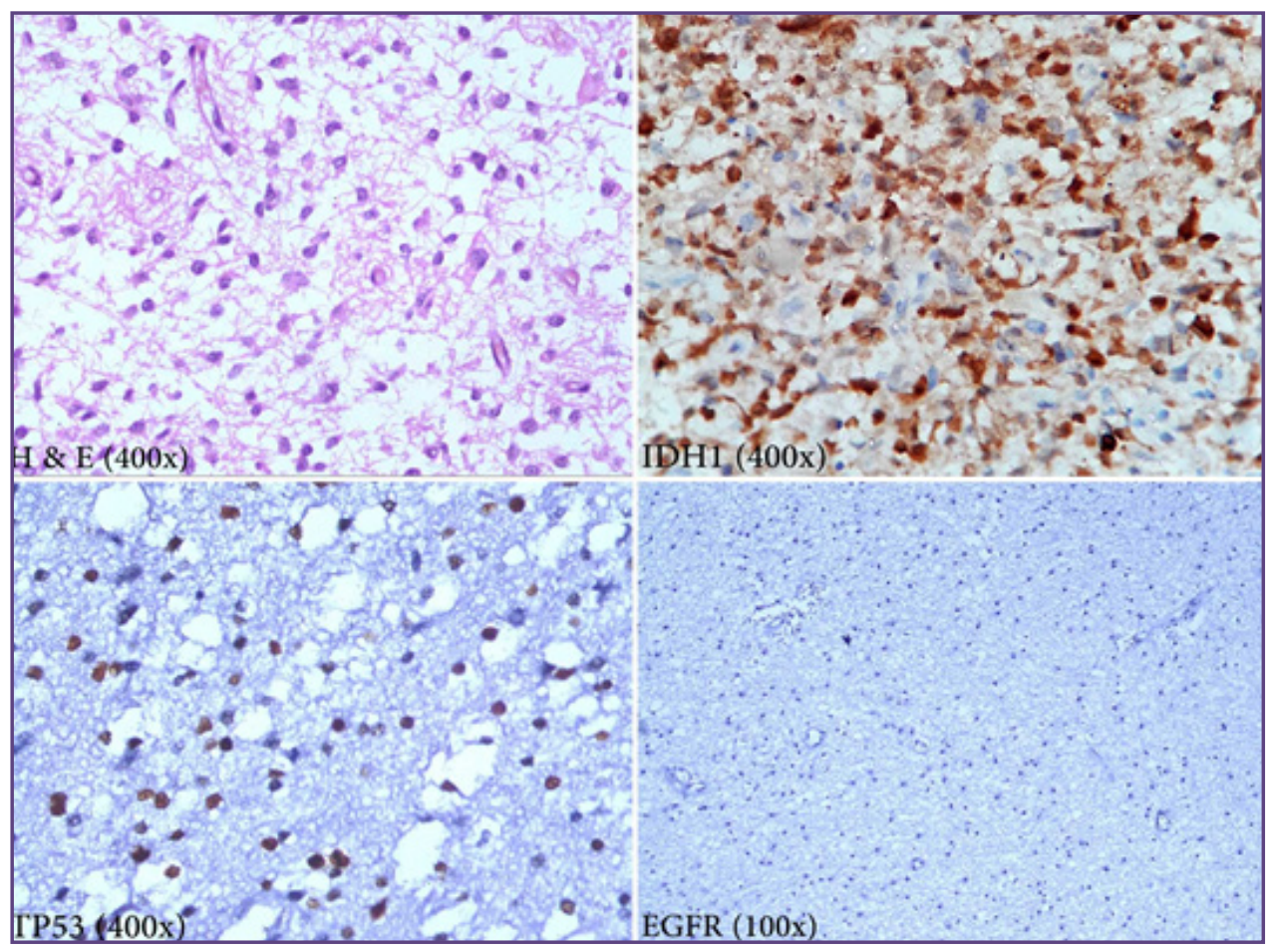

Fig. 3: Photomicrograph showing H \& E stained section of Diffuse Astrocytoma (WHO grade II) with IDH1R132H, TP53 and EGFR protein expression. IDH1R132H (cytoplasmic) and TP53 (Nuclear) are positive in tumour cells. EGFR protein expression is negative.

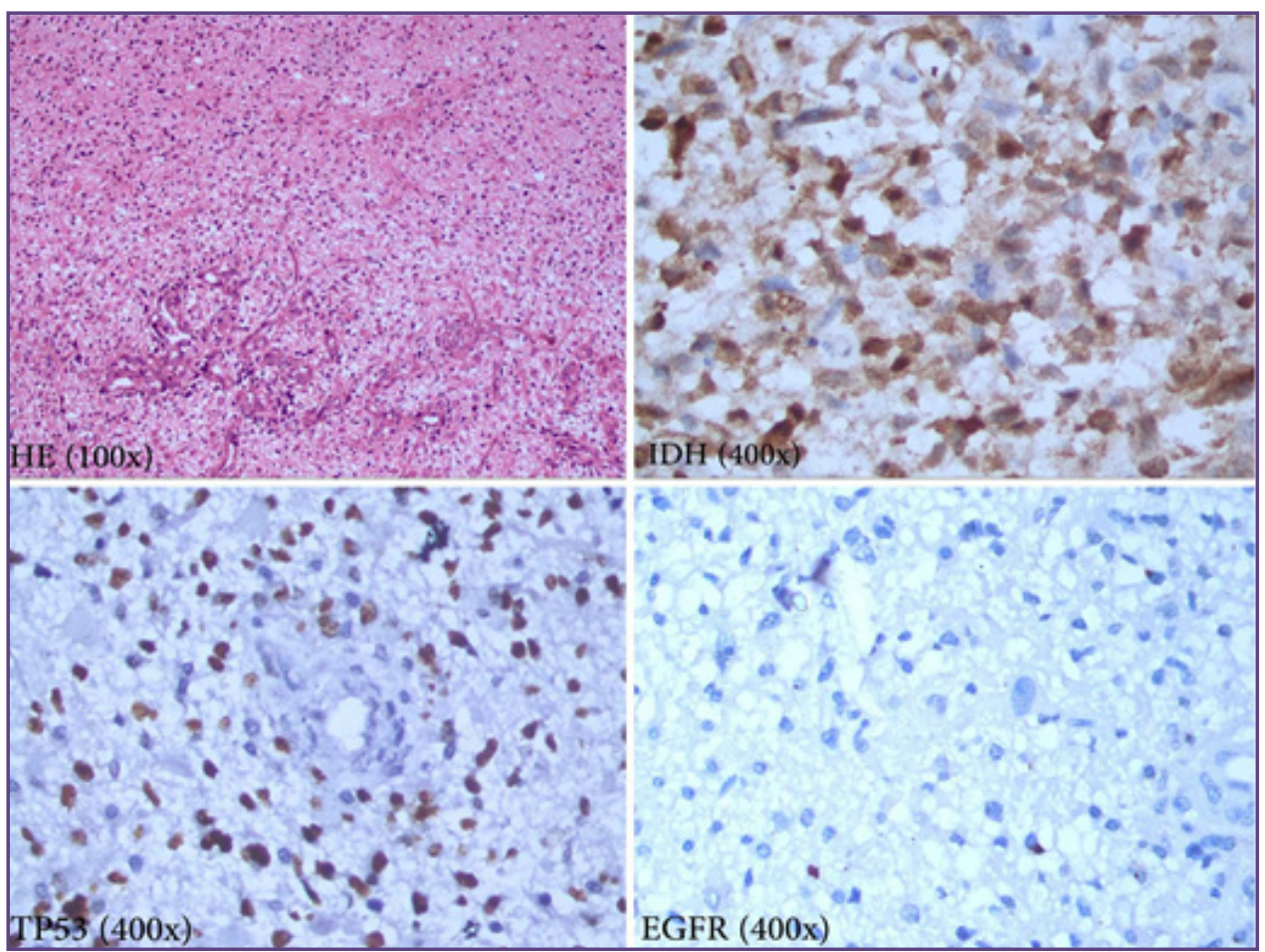

Fig. 4: Photomicrograph showing H \& E stained section of Anaplastic Astrocytoma (WHO grade III) with IDH1R132H, TP53 and EGFR protein expression. IDH1R132H (cytoplasmic) and TP53 (Nuclear) are positive in tumour cells. EGFR protein expression is negative. 


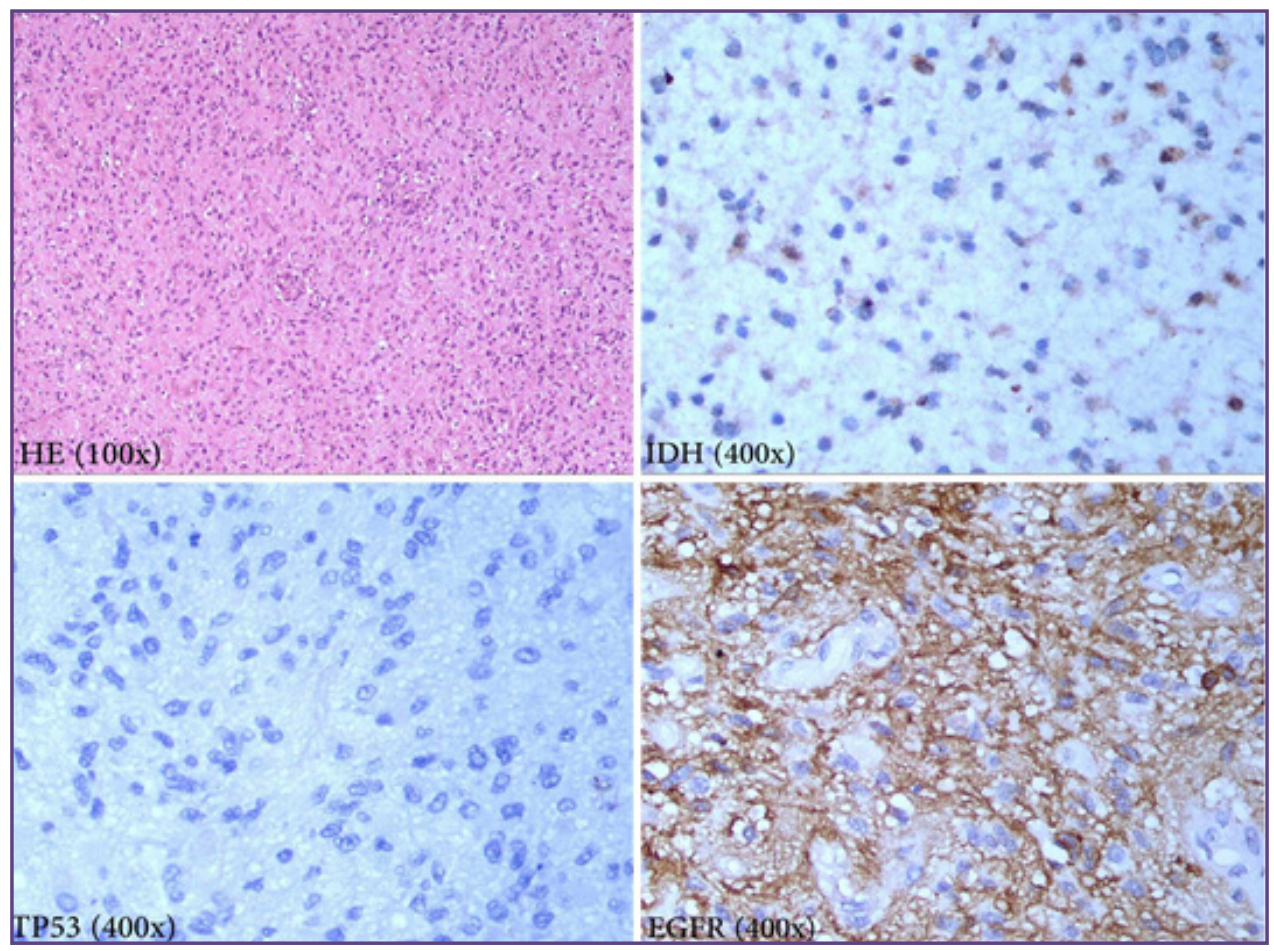

Fig. 5: Photomicrograph showing H \& E stained section of Primary GBM (WHO grade IV) with IDHR132H, TP53 and EGFR protein expression. EGFR protein overexpression (cytoplasmic) is seen in tumour cells. IDH1R132H and TP53 expression is negative.

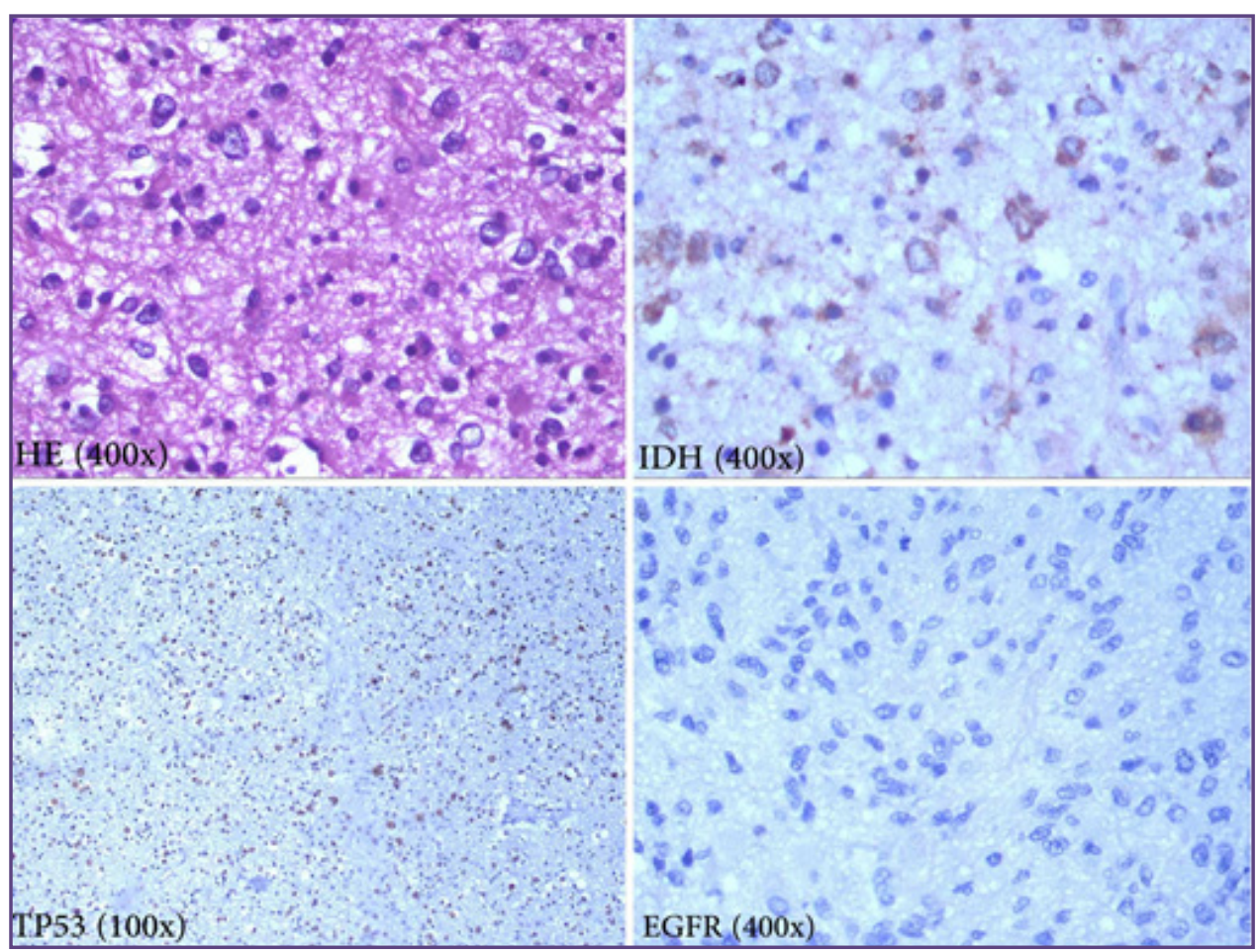

Fig. 6: Photomicrograph showing H \& E stained section of Secondary GBM (WHO grade IV) with IDHR132H, TP53 and EGFR protein expression. IDHR132H (cytoplasmic) and TP53 (Nuclear) are positive in tumour cells. EGFR protein expression is negative. 
In previous studies 59-90 \% of diffuse astrocytoma, 28-82 $\%$ of anaplastic astrocytoma and $70-75 \%$ of secondary GBM showed IDH1R132H positivity. Whereas IDH1 mutations are less frequent in primary $\operatorname{GBM}(<10 \%)$ and rare in PA. ${ }^{[3,16]}$

In the present study, we have studied the immunohistochemical expression ofIDH1R132Hmutations across various grades of astrocytomas and found that $13 \%$ of pilocytic astrocytoma, $52 \%$ of DA, $85 \%$ of AA, $80 \%$ of secondary GBM and $21 \%$ of primary GBM expressed IDH1R132H. We found IDH mutations are significantly associated with astrocytoma grade.

p53 is a tumour suppressor gene which is mutated in a vast majority of human tumours. ${ }^{[17]}$ Mutated p53 encodes for a mutated nuclear protein which cannot be degraded easily and accumulates in the nucleus. ${ }^{[18]}$ This mutated TP53 can be detected by IHC and so overexpression of p53 is considered to be a surrogate marker for p53 mutation. In our study, it was observed that TP53 expression was significantly higher in grade II (71\%) and grade III (86\%) astrocytomas. Grade I astrocytomas arise from different molecular alterations and thus do not exhibit p53 mutation (figure 2). Among grade IV astrocytomas the expression was variable among primary GBM $(8.1 \%)$ and secondary GBM (26.6\%). A significant association was found between the grade of tumour and TP53 expression ( $\mathrm{p}$ value of $<0.001$ using Fisher's exact test). Tumours with mutated p53 cannot tolerate genotoxic stress and as a result, undergo apoptosis when chemotherapeutic drugs and radiotherapy is used against them. ${ }^{[19]}$

Previous studies have shown that the frequency of EGFR gene amplification increases with increasing tumour grade.

${ }^{[20]}$ EGFR amplification is rare in grade II astrocytomas and is seen in only $4 \%$ of cases in some studies. Glioblastomas show the highest expression of EGFR among all four grades and EGFR amplification is seen in around $60 \%$ of cases. ${ }^{[21,22]}$ In our study, EGFR over-expression was not seen in any case of PA. Only $2 / 21$ cases $(9.5 \%)$ of DA showed EGFR over-expression. Primary GBM show the highest percentage of EGFR protein expression with 26/37 cases showing EGFR positivity (70.3\%). Secondary GBM, in contrast, show an expression profile similar to low grade gliomas with only $2 / 15$ cases showing EGFR over-expression (13.3\%). In the present study, EGFR overexpression was associated with the higher-grade gliomas, suggesting that EGFR overexpression was associated with tumour aggressiveness and invasion.

Association of IDHR132H with TP53 and EGFR Protein Expression: The results of our study show that IDHR132H mutation is significantly associated with TP53 expression.
On the other hand, a reverse relationship was found between IDH1 mutation and EGFR protein expression. TP53 and IDH1 expression are found in grade II (figure 3 ) and III (figure 4) astrocytomas and younger age group patients. ${ }^{[4]}$ These tumours also acquire mutation of TP53 gene along their natural history and thus both mutations are often found in these group of tumours. The inverse relationship between IDH1 mutation and EGFR expression and the preferential expression of EGFR in glioblastomas shows that glioblastomas arise from a genetic mechanism which is different from lower grade gliomas (figure 5). This view is further strengthened by the fact that secondary glioblastomas arise from lower grade astrocytomasand show an immunohistochemical profile similar to grade II and III astrocytomas (figure 6).

To our knowledge studies showing a functional relationship between IDH1, p53 and EGFR expression in various grades of astrocytomas are few. Our study shows a strong association between IDH1R132Hwith TP53 and an inverse association with EGFR protein expression. ${ }^{[23]}$ Other studies probing new pathways involved in gliomagenesis are underway. A better understanding of these mutations will help investigators develop newer targeted therapies against high-grade gliomas, which as of date are incurable. Targeted therapies which act as competitive antagonists of mutant Isocitrate dehydrogenase enzyme are in development. ${ }^{[24]}$ Glioma patients harbouring IDH mutation may benefit from these therapies in future. ${ }^{[25]}$

\section{Conclusion}

IDHR132H and TP53 mutations are seen in the majority of DA, AA and secondary GBM. Primary GBM, in contrast, were found to overexpress EGFR protein and thus arise from a molecular pathway that is different from that of lower grade gliomas.IDHR132H and EGFR protein expression was found to be mutually exclusive and there is a significant inverse association between these two IHC markers.

Further studies need to be done to better understand the pathogenesis of gliomas so that new molecular targets are identified against which targeted therapy can be developed to treat high-grade gliomas which are incurable and have a poor prognosis and survival despite treatment.

\section{References}

1. CBTRUS - 2016 CBTRUS Fact Sheet [Internet]. Available from: http://www.cbtrus.org/factsheet/factsheet.html

2. IARC Publications Website - WHO Classification of Tumours of the Central Nervous System [Internet]. Available from: http://publications.iarc.fr/Book-And-Report-Series/ Who-Iarc-Classification-Of-Tumours/Who-ClassificationOf-Tumours-Of-The-Central-Nervous-System-2016 
3. Ichimura K, Ohgaki H, Kleihues P, Collins VP. Molecular pathogenesis of astrocytic tumours. $\mathrm{J}$ Neurooncol. 2004;70(2):137-60.

4. Ohgaki H, Kleihues P. The Definition of Primary and Secondary Glioblastoma. Clin Cancer Res. 2013;19(4):764-72.

5. Huang $\mathrm{PH}, \mathrm{Xu} \mathrm{AM}$, White FM. Oncogenic EGFR signaling networks in glioma. Sci Signal. 2009;2(87):re6.

6. Heimberger AB, Crotty LE, Archer GE, Hess KR, Wikstrand $\mathrm{CJ}$, Friedman AH, et al. Epidermal growth factor receptor VIII peptide vaccination is efficacious against established intracerebral tumours. Clin Cancer Res. 2003;9(11):4247-54.

7. Watanabe $\mathrm{T}$, Nobusawa $\mathrm{S}$, Kleihues $\mathrm{P}$, Ohgaki $\mathrm{H}$. IDH1 mutations are early events in the development of astrocytomas and oligodendrogliomas. Am J Pathol. 2009;174(4):1149-53.

8. Kim SY, Park J-W. Cellular defense against singlet oxygeninduced oxidative damage by cytosolic NADP+-dependent isocitrate dehydrogenase. Free Radic Res. 2003;37(3):309-16.

9. Lee SM, Koh H-J, Park D-C, Song BJ, Huh T-L, Park J-W. Cytosolic NADP(+)-dependent isocitrate dehydrogenase status modulates oxidative damage to cells. Free Radic Biol Med. 2002;32(11):1185-96.

10. Yan H, Parsons DW, Jin G, McLendon R, Rasheed BA, Yuan $\mathrm{W}$, et al. IDH1 and IDH2 mutations in gliomas. N Engl J Med. NIH Public Access; 2009;360(8):765-73.

11. Bleeker FE, Atai NA, Lamba S, Ard J, Rijkeboer D, et al. The prognostic IDH1 R132 mutation is associated with reduced NADP + -dependent IDH activity in glioblastoma.

12. Zhao S, Lin $\mathrm{Y}, \mathrm{Xu}$ W, Jiang W, Zha Z, Wang P, et al. Gliomaderived mutations in IDH1 dominantly inhibit IDH1 catalytic activity and induce HIF-1alpha. Science. NIH Public Access; 2009;324(5924):261-5.

13. Liu X-Y, Gerges N, Korshunov A, Sabha N, Khuong-Quang D-A, Fontebasso AM, et al. Frequent ATRX mutations and loss of expression in adult diffuse astrocytic tumours carrying IDH1/IDH2 and TP53 mutations. Acta Neuropathol. 2012;124(5):615-25.

14. Nobusawa S, Watanabe T, Kleihues P, Ohgaki H. IDH1 Mutations as Molecular Signature and Predictive Factor of Secondary Glioblastomas. Clin Cancer Res. 2009;15(19).
15. Ohgaki H, Kleihues P. Genetic Pathways to Primary and Secondary Glioblastoma. Am J Pathol. 2007;170(5):1445-53.

16. Ichimura K, Pearson DM, Kocialkowski S, Bäcklund LM, Chan R, Jones DTW, et al. IDH1 mutations are present in the majority of common adult gliomas but rare in primary glioblastomas. Neuro Oncol [Internet]. Oxford University Press; 2009;11(4):341-7.

17. Muller PAJ, Vousden KH. p53 mutations in cancer. Nat Cell Biol. Nature Research; 2013;15(1):2-8.

18. Louis DN. The $\mathrm{p} 53$ gene and protein in human brain tumours. J Neuropathol Exp Neurol. 1994;53(1):11-21.

19. Bourdon JC, Laurenzi V De, Melino G, Lane D. p53: 25 years of research and more questions to answer. Cell Death Differ. Nature Publishing Group; 2003;10(4):397-9.

20. Nishikawa R, Ji XD, Harmon RC, Lazar CS, Gill GN, Cavenee WK, et al. A mutant epidermal growth factor receptor common in human glioma confers enhanced tumourigenicity. Proc Natl Acad Sci U S A. 1994;91(16):7727-31.

21. Smith JS, Tachibana I, Passe SM, Huntley BK, Borell TJ, Iturria N, et al. PTEN mutation, EGFR amplification, and outcome in patients with anaplastic astrocytoma and glioblastoma multiforme. $\mathrm{J}$ Natl Cancer Inst. 2001;93(16):1246-56.

22. Hatanpaa KJ, Burma S, Zhao D, Habib AA. Epidermal growth factor receptor in glioma: signal transduction, neuropathology, imaging, and radioresistance. Neoplasia. Neoplasia Press; 2010;12(9):675-84.

23. Watanabe K, Tachibana O, Sata K, Yonekawa Y, Kleihues P, Ohgaki H. Overexpression of the EGF receptor and p53 mutations are mutually exclusive in the evolution of primary and secondary glioblastomas. Brain Pathol. 1996;6(3):217-23-4.

24. Popovici-Muller J, Saunders JO, Salituro FG, Travins JM, Yan S, Zhao F, et al. Discovery of the First Potent Inhibitors of Mutant IDH1 That Lower Tumour 2-HG in Vivo. ACS Med Chem Lett. American Chemical Society; 2012;3(10):850-5.

25. Carlsson SK, Brothers SP, Wahlestedt C. Emerging treatment strategies for glioblastoma multiforme. EMBO Mol Med. Wiley-Blackwell; 2014;6(11):1359-70.

*Corresponding author:

Dr Shashank Mishra, Resident, Department of Pathology, Armed Forces Medical College, Pune, India

Email: drshashankmishra@hotmail.com

Date of Submission : 03.04.2017

Date of Acceptance : 14.06.2017

Financial or other Competing Interests: None.

Date of Publication : 26.10.2017 